\title{
3 Research Soure \\ PD-L1 Mediates Triple Negative Breast Cancer Evolution by Regulating TAM/M2 Polarization
}

ziqi meng

Yanbian University Medical College

Rui Zhang

Yanbian University Medical College

Xuwei Wu

Yanbian University Medical College

Meihua Zhang

Yanbian University Hospital

Tiefeng Jin ( $\nabla$ jintf@ybu.edu.cn )

Yanbian University Medical College

\section{Research Article}

Keywords: PD-L1, TAMs, metastasis, angiogenesis, STAT3, TNBC

Posted Date: February 24th, 2022

DOI: https://doi.org/10.21203/rs.3.rs-1357366/v1

License: (c) (1) This work is licensed under a Creative Commons Attribution 4.0 International License.

Read Full License 


\section{Abstract}

\section{Aim}

This study aimed to explore the role of aPD-L1 (PD-L1 inhibitor) in triple-negative breast cancer (TNBC) cells metastasis and angiogenesis by regulating tumor-associated macrophages (TAMs) polarization and its possible molecular mechanism.

\section{Methods}

The effects of aPD-L1 on tumor metastasis and TAMs polarization were confirmed both in vitro and in vivo. The expression of M2-type macrophages (TAM/M2) and M1-type macrophages (TAM/M1) surface markers were detected by flow cytometry, western blot and IF staining assays. The effects of aPD-L1 on the proliferation and apoptosis of TAM/M2 were detected by MTT and flow cytometry assays. The effects of APD-L1 on the migration capability of TNBC cells were detected by wound healing and transwell assays. The effects of angiogenesis were detected by endothelial tube formation and western blot assays. The effects of aPD-L1 on epithelial-mesenchymal transition (EMT) process and stemness of TNBC cells were detected by western blot and IF staining assays. The expression and location of STAT3 and p-STAT3 proteins in cells were detected by western blot and IF staining assays.

\section{Results}

In this study, we demonstrated that aPD-L1 significantly inhibited IL-13-induced TAM/M2 polarization in vitro. aPD-L1 inhibited EMT process and stemness of TNBC cells mediated by reversing TAM/M2 polarization, thus inhibiting the migration and angiogenesis of TNBC cells. aPD-L1 prevented STAT3 phosphorylation and nuclear translocation, resulting in TAM/M2 polarization arrest. APD-L1 reduced the number of lung metastases without affecting tumor growth in vivo. aPD-L1 reduced the expression of TAM/M2, EMT, stemness, and vascular markers in tumor tissues.

\section{Conclusions}

Taken together, these data suggest that aPD-L1 plays a vital role in anti-metastasis and anti-angiogenesis of TNBC in vitro and in vivo by inhibiting TAM/M2 polarization.

\section{Background}

Breast cancer is the most common cancers and the main cause of cancer deaths in women ${ }^{[1]}$. According to the reports, breast cancer accounts for approximately $11.7 \%$ of global cancer cases and $15.5 \%$ of all female cancer deaths ${ }^{[2]}$. Breast cancer is divided into four subtypes according to its molecular 
phenotypes: Luminal A, Luminal B, human epidermal growth factor receptor-2 (HER2) and TNBC ${ }^{[3]}$. TNBC accounts for about $15 \%$ of all breast cancers ${ }^{[4]}$ TNBC is characterized by obvious heterogeneity, low age of onset, late stage, high risk of visceral metastasis, high tumor invasiveness, and high histological grade ${ }^{[5]}$. However, TNBC lacks specific molecular targets and metastasis occurs in the early stage. Traditional treatment methods are ineffective and there is no effective treatment strategy.

Tumor microenvironment (TME) plays a decisive role in the occurrence and development of cancers ${ }^{[6]}$. Tumor cells promote the evolution by changing TME to create favorable conditions for their own survival. TAMs are important immune cells in the TME, accounting for $50-80 \%$ of mesenchymal cells ${ }^{[7]}$. TAMs have strong plasticity and polarizes into different phenotypes under the stimulation of TME and cytokines. TAMs can be divided into macrophages activated by the classical pathway TAM/M1 and the alternative pathway TAM/M2 according to their different polarization modes. TAM/M1 mainly showed tumor inhibition, while TAM/M2 showed more tumor promotion ${ }^{[8]}$. About $90 \%$ of breast cancer deaths are caused by metastasis ${ }^{[9]}$. Cytokines in the TME interact with tumor cells and play an important role in the process of tumor metastasis ${ }^{[10]}$. TAM/M2 is associated with poor prognosis of breast cancer, colorectal cancer and gastric cancer ${ }^{[11-13]}$. EMT is associated with tumor metastasis and poor prognosis, and is considered to be the mechanism of tumor metastasis ${ }^{[14]}$. Study reported that TAMs enhance metastasis of colorectal cancer cells by inducing EMT process ${ }^{[15]}$. Cancer stem cells are self-regenerative and high carcinogenic potential, which are considered to be related to chemotherapy resistance, metastasis and recurrence ${ }^{[16]}$. TAMs-derived cytokines enhance the stemness of cancer through the EMT process ${ }^{[17]}$. TAM/M2 induces angiogenesis and secrete pro-angiogenic factors such as vascular endothelial growth factor (VEGF), degrade tumor extracellular matrix and help tumor cells escape immune supervision to promote tumor metastasis, immunosuppression, drug resistance, and provide nutrition and metastasis pathways for tumor growth ${ }^{[18]}$. Therefore, reversing the polarization of TAM/M2, reducing its recruitment, and blocking the tumor-promoting function of TAM/M2 may be a new anti-tumor strategy.

Programmed death receptor-1 (PD-1) is an inhibitory co-receptor that binds to programmed death ligand-1 (PD-L1) to effectively inhibit T cell activity, thereby reducing $T$ cell recognition to tumor cells and enabling tumor cells to evade immune supervision. The expression of PD-1 and PD-L1 in the TNBC patients tissues are higher than other molecular types of breast cancer ${ }^{[19]}$. PD-L1 inhibitors have a long-lasting effect on advanced TNBC ${ }^{[20]}$. Study showed that tumor drug resistance seriously restricted the later efficacy of PD1/PD-L1 inhibitors ${ }^{[21]}$. The expression of PD-L1 in TME of tumor cells and host immune cells assist tumor cells immune escape, and monitoring the total expression of PD-L1 in tumor tissues is more significant than the expression of tumor cells ${ }^{[22]}$. The expression of PD-L1 plays a decisive role in host immune cells, rather than tumor cells ${ }^{[23]}$. Therefore, the expression of PD-L1 in TAMs may play a key role in tumor progression. These findings suggest that TNBC may induce TAM/M2 polarization and promote tumor evolution. However, whether APD-L1 inhibits TNBC malignant progression by reversing TAM/M2 polarization has not been reported. 


\section{Material And Methods \\ 2.1 Cell culture}

Yanbian University Cancer Research Center supplied the human TNBC cell lines (MDA-MB-231 and Hs578T), human umbilical vein endothelial cells (HUVECs), and mouse macrophages (RAW264.7). All cells were cultured in RPMI-DMEM containing 10\% FBS and 1\% streptomycin-penicillin $(100 \mathrm{U} / \mathrm{mL})$. Cells were incubated at $37^{\circ} \mathrm{C}$ in an atmosphere of $5 \% \mathrm{CO}_{2}$.

\subsection{Flow cytometry assay}

Cells $\left(5 \times 10^{5}\right)$ suspension was washed twice with cell staining buffer. Fix the cells with fixation buffer, and break the cell membrane with permeabilization buffer. Cells were then stained with CD68-FITC (mouse), CD206-PE (mouse), and CD86-APC (mouse) antibodies (Biolegend, CA, USA). Cells were suspended with $500 \mu \mathrm{L}$ cell staining buffer and examined with a BD Accuri C6 flow cytometer (BD Biosciences, CA, USA). All tests are controlled by homologous isotype control antibodies.

\subsection{Western blot assay}

Cells were collected and appropriate amount of RIPA lysate (RIPA lysate: PMSF = 100:1) was added according to the number of cells. The protein concentration was determined and quantified using BSA kit (Roche, Basel, Switzerland). Proteins were separated by SDS-polyacrylamide gel electrophoresis and transferred to PVDF membrane (Millipore, Bedford, MA). The membranes were incubated with the primary antibody at $4^{\circ} \mathrm{C}$ overnight and then the secondary antibody were incubated at RT for $1 \mathrm{~h}$. Enhanced chemiluminescence detects antibody signals by BioRad system (Hercules, CA, USA) and collects images.

\subsection{Immunofluorescence (IF) staining}

Slides with cells on the 6-well plates were fixed with methanol for $15 \mathrm{~min}$ and permeated with $0.5 \%$ Triton X-100 (CWBIO, Beijing, China) for 10 min. Then, blocked with 3\% BSA (Solarbio, Beijing, China) for $2 \mathrm{~h}$. Cells were incubated with primary antibody in $3 \% \mathrm{BSA}$ at $4{ }^{\circ} \mathrm{C}$ overnight and incubated with Alexa Fluor 488 Goat Anti-Rabbit IgG or Alexa Fluor® 568 goat anti-mouse IgG (1:400; Invitrogen, CA, USA) for $1 \mathrm{~h}$. Cells were counterstained with DAPI and imaged by Leica SP5II confocal microscope.

\subsection{ELISA}

ELISA was performed according to the instructions. The concentrations of IL-10 (mouse) and IL-12 (mouse) in cell culture supernatants were determined using the kit (Mlbio, Shanghai China).

\subsection{MTT assay}

Cells $\left(5 \times 10^{3}\right)$ suspension was prepared and incubated on 96-well plates for $24 \mathrm{~h}$. Conditional medium or drugs were added and incubated for $0,24,48$ and $72 \mathrm{~h}$, respectively. Add $100 \mu \mathrm{L}$ MTT reagent $(1 \mathrm{mg} / \mathrm{mL})$ to each well and incubate for $4 \mathrm{~h}$ under the same conditions. Then, add $100 \mu \mathrm{L}$ DMSO to each well and 
the absorbance value (OD) at $490 \mathrm{~nm}$ was measured. Five wells per group at least were analyzed and repeated three times.

\subsection{Apoptosis assay}

Cells $\left(1 \times 10^{6}\right)$ suspension was washed twice with binding buffer. Cells were stained using the Annexin VFITC Apoptosis Detection Kit (BD Biosciences, CA, USA) according to the instruction. Cells were analyzed with a BD Accuri C6 flow cytometer.

\subsection{Cell morphology}

RAW264.7 cells with a 30\% fusion rate were incubated on 6-well plates for $12 \mathrm{~h}$ and added with IL-13 and/or APD-L1 for $48 \mathrm{~h}$. Morphological changes of cells were observed under a microscope and images were collected.

\subsection{Conditioned Medium (CM) Preparation}

RAW264.7 cells were treated with TAM/M2 inducing factor Interleukin-13 (30 ng/mL) (IL-13, Biolegend,

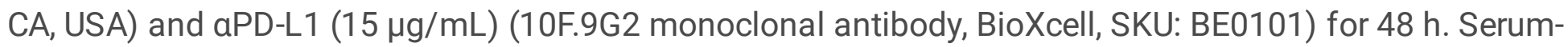
free medium was cultured for $24 \mathrm{~h}$, and three CM (RAW264.7, IL-13/RAW264.7 and IL-13 + aPDL1/RAW264.7) were prepared. The CM can be directly used for assays or stored at $-80^{\circ} \mathrm{C}$. The CM was filtered through a screen and added with $2 \%$ FBS.

\subsection{Migration assay}

Cells $\left(5 \times 10^{4}\right)$ in $100 \mu \mathrm{L}$ containing $10 \%$ serum RPMI-DMEM were seeded onto the upper chambers. The bottom chambers were filled with culture medium containing 10\% FBS RPMI-DMEM, and the cells were incubated at $37^{\circ} \mathrm{C}$ for $6 \mathrm{~h}$. Then, the upper chambers were replaced with different groups of CM. Cells passing through the subsurface of the filtration membrane were fixed with $4 \%$ paraformaldehyde for 20 min and then stained with crystal violet for $20 \mathrm{~min}$. Three fields (200x) were randomly selected under the microscope to collect images. Image J software ( $\mathrm{NIH}$, Bethesda, MD, USA) counted the number of cells in each field.

\subsection{Wound healing assay}

Cells were incubated on 6-well plates for $24 \mathrm{~h}$. Scratch the wound vertically with a $200 \mu \mathrm{L}$ pipette tip and wash three times with PBS to remove dead cells in the well. The CM (containing IL-13 and/or aPD-L1) was incubated at $37^{\circ} \mathrm{C}$ in an atmosphere of $5 \% \mathrm{CO}_{2}$. Take pictures at 0,12 , and $24 \mathrm{~h}$.

\subsection{Endothelial tube formation assay}

The matrigel (BD Biosciences, San Diego, CA, USA) and RPMI-DMEM were diluted 1:1 in 96-well plates and solidify at $37^{\circ} \mathrm{C}$ for $4 \mathrm{~h}$. HUVECs $\left(2 \times 10^{4}\right)$ were incubated in 2:1 diluted $\mathrm{CM}$ and culture medium at $37^{\circ} \mathrm{C}$ for $4 \mathrm{~h}$. Capillary structure was collected under the microscope. 


\subsection{Establish cell co-culture system}

RAW264.7 cells $\left(5 \times 10^{4}\right)$ in $600 \mu \mathrm{L}$ containing $10 \%$ serum RPMI-DMEM were seeded onto the bottom chambers. MDA-MB-231 or Hs $578 \mathrm{~T}$ cells $\left(1 \times 10^{5}\right)$ in $100 \mu \mathrm{L}$ containing $10 \%$ serum RPMI-DMEM were seeded onto the upper chambers, and the cells were incubated at $37^{\circ} \mathrm{C}$ for $6 \mathrm{~h}$. The supernatant was harvested for ELISA assay, and RAW264.7 cells were harvested for western blot and flow cytometry assays.

\subsection{Animal studies}

4T1 cells $\left(5 \times 10^{5}\right)$ were injected into subcutaneous of 5 -week-old BALB/c female mice to establish tumor model. Tumor size was measured every 3 days and tumor volume was calculated using the formula length $\times$ width $^{2} \times 0.5$. The animals were sacrificed after 25 days of aPD-L1 treatment. For detecting lung metastases, $4 \mathrm{~T} 1$ cells $\left(1 \times 10^{5}\right)$ were injected into the tail vein of $\mathrm{BALB} / \mathrm{c}$ female mice. The lungs were collected and the surface nodules were counted. The tumor and lung tissues were fixed with formalin and sectionalized. The expression of the markers was confirmed by IHC staining. All experiments were performed in accordance with the procedures of the Animal Ethics Committee of the Yanbian University.

\subsection{Immunohistochemistry (IHC) staining}

Tumor and lung tissue sections were dewaxed and dehydrated. Then, the tissue sections were repaired with citrate buffer $\left(\mathrm{pH} \mathrm{7.0)}\right.$ at $80^{\circ} \mathrm{C}$ for 20 min. The tissue sections were incubated with $3 \% \mathrm{H}_{2} \mathrm{O}_{2}$ at RT for $30 \mathrm{~min}$. The tissue sections were incubated with primary antibody at $4{ }^{\circ} \mathrm{C}$ overnight. After incubation with secondary antibody at RT for $1 \mathrm{~h}$. Slide immunostaining was advanced using DAB and counterstained with hematoxylin.

\subsection{Statistical analysis}

GraphPad Prism 8.0 software (GraphPad, La Jolla, CA, USA) was used for statistical analysis. T-test was used to compare the mean values of two samples. One-way ANOVA was used to compare the mean values of multiple groups. All experimental data were repeated for three times and their mean values were taken as mean \pm standard deviation. Depending on the experiment, the significance was $* P<0.05$, $* \star P<$

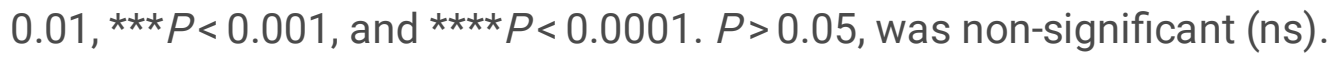

\section{Results}

\section{1 aPD-L1 reverses TAM/M2 polarization.}

RAW264.7 cells were treated with continuous concentration of TAM/M2 inducing factor IL-13 for $48 \mathrm{~h}$. IF staining, western blot, and ELISA analysis confirmed that IL-13 promoted the expression of TAM/M2 marker CD206 in the RAW264.7 cells cytoplasm, and the secretion of IL-10 in a dose-dependent manner. IL-13 at the concentration of $30 \mathrm{ng} / \mathrm{mL}$ was selected for further investigation (Fig. 1A-D). RAW264.7 cells 
were treated with continuous concentrations of aPD-L1 for 24, 48, and $72 \mathrm{~h}$. MTT analysis confirmed that aPD-L1 at concentrations below $15 \mu \mathrm{g} / \mathrm{mL}$ did not affect the proliferation of RAW264.7 cells. aPD-L1 at the concentration of $15 \mu \mathrm{g} / \mathrm{mL}$ was selected for further investigation (Fig. 1E).

To investigate the function of APD-L1 on TAM/M2 cells. We first verified the expression of PD-L1 in TAM/M2 by western blot assay, and the results confirmed that the expression of PD-L1 was upregulated in TAM/M2 cells and aPD-L1 downregulated the expression of PD-L1 (Fig. 1F). Then, we further confirmed that IL-13 $(30 \mathrm{ng} / \mathrm{mL})$ and/or aPD-L1 $(15 \mu \mathrm{g} / \mathrm{mL})$ had no effect on proliferation and apoptosis capability of RAW264.7 cells by MTT and flow cytometry assays. (Fig. 1G-H). Flow cytometry, western blot and ELISA analysis confirmed that APD-L1 inhibited the expression of CD206 and the secretion of IL10 induced by IL-13. However, the expression of TAM/M1 marker CD86 and the secretion of IL-12 were not affected by IL-13 and/or aPD-L1 (Fig. 1I-K). To further investigate the effects of aPD-L1 on TAM/M2 polarization, the changes of cell morphology were observed through a microscope. IL-13 stimulated RAW264.7 cells to change from round or oval to long spindle shape, while aPD-L1 inhibited cell extension (Fig. 1L). These data suggested that aPD-L1 inhibits TAM/M2 polarization.

\section{2 aPD-L1 inhibits TAM/M2-induced the migration and angiogenesis of TNBC cells.}

RAW264.7 cells were treated with IL-13 and/or aPD-L1 for $48 \mathrm{~h}$, and supernatant was replaced with serum-free medium for $24 \mathrm{~h}$ and then collected as CM (Fig. 2A). To investigate the effects of aPD-L1 on the interaction between TAM/M2 and TNBC cells, we confirmed that the CM had no effect on the proliferation and apoptosis of TNBC cells by MTT and flow cytometry assays (Fig. 2B-C). Then, wound healing and transwell analysis confirmed that MDA-MB-231 and Hs578T cells treated with the CM of IL13/RAW264.7 promoted the migration capability, while the CM of IL-13 + aPD-L1/RAW264.7 reversed this phenomenon (Fig. 2D-E).

Neovascularization in tumor tissues is an important condition for malignant tumor metastasis, which is regulated by various chemokines in TME ${ }^{[24]}$. To investigate the effects of APD-L1 on angiogenesis through TAM/M2 polarization in vitro. We performed endothelial tube formation analysis, and the results confirmed that HUVECs treated with the CM of IL-13/RAW264.7 promoted the microtubule formation, while the CM of IL-13 + aPD-L1/RAW264.7 reversed this phenomenon (Fig. 2F). We then performed western blot analysis, and the results confirmed that MDA-MB-231 and Hs578T cells treated with the CM of IL-13/RAW264.7 upregulated the expression of VEGF, MMP2, and MMP9, while the CM of IL-13 + aPDL1/RAW264.7 reversed this phenomenon (Fig. 2G). These data suggested that aPD-L1 inhibits TNBC cells migration and angiogenesis induced by TAM/M2 polarization.

\section{3 aPD-L1 inhibits TAM/M2-induced EMT process and stemness of TNBC cells.}

To investigate whether APD-L1 inhibits the EMT process of TNBC cells by regulating TAMs polarization. We performed western blot and IF staining analysis, and the results confirmed that MDA-MB-231 and 
Hs578T cells treated with the CM of IL-13/RAW264.7 upregulated the expressions of mesenchymal markers N-Cadherin, Vimentin, Slug, Twist, and downregulated the expressions of epithelial markers ECadherin, ZO-1. However, MDA-MB-231 and Hs578T cells treated with the CM of IL-13 + aPD-

L1/RAW264.7 eliminated this phenomenon (Fig. 3A-B).

Tumor cells expressed high levels of stem cells surface markers after EMT progression, indicating that these cells have become stem cells ${ }^{[25]}$. To investigated whether APD-L1 inhibits the stemness of TNBC cells by regulating TAM/M2 polarization. We performed western blot and IF staining analysis, and the results confirmed that MDA-MB-231 and Hs578T cells treated with the CM of IL-13/RAW264.7 upregulated the expressions of stemness markers CD44, Oct4, Nanog, Bmi1, and Sox2. However, MDAMB-231 and Hs578T cells treated with the CM of IL-13 + aPD-L1/RAW264.7 eliminated this phenomenon (Fig. 3C-D). These data suggested that aPD-L1 inhibits the EMT process and stemness of TNBC cells by reversing TAM/M2 polarization, thereby inhibiting TNBC metastasis and angiogenesis.

\section{4 aPD-L1 reverses TAM/M2 polarization in co-culture system.}

We identified the effects of TAM/M2 polarization on TNBC cells. Thus, we established co-culture system of TAMs and TNBC cells to simulate the interaction relationship in real TME. Six groups: 1) Negative control group: RAW264.7 cells were cultured separately; 2) Positive control group: RAW264.7 cells were treated with IL-13 to construct TAM/M2; 3) Co-culture group 1: RAW264.7 was co-cultured with MDA-MB231 cells; 4) Co-culture dope group 1: RAW264.7 cells were co-cultured with MDA-MB-231 cells by aPD-L1 treatment; 5) Co-culture group 2: RAW264.7 was co-cultured with Hs578T cells. 6) Co-culture dope group 2: RAW264.7 cells were co-cultured with Hs578T cells by aPD-L1 treatment (Fig. 4A). As expected, western blot analysis confirmed that the percentage of $\mathrm{CD}^{206^{+}} / \mathrm{CD}^{+} 8^{+}$(TAM/M2) cells in RAW264.7 cocultured with TNBC cells was upregulated, while aPD-L1 downregulated the percentage of CD206 ${ }^{+} / \mathrm{CD}^{2} 8^{+}$ cells. However, the percentage of $\mathrm{CD} 86^{+} / \mathrm{CD} 68^{+}$(TAM/M1) cells did not change significantly in the coculture system with or without aPD-L1 (Fig. 4B-C). To further confirm our results, ELISA analysis confirmed that the secretion of IL-10 in positive control group, co-culture group 1, and co-culture group 2 was increased, while APD-L1 reversed this phenomenon. However, the secretion of IL-12 did not affect in the co-culture system with or without aPD-L1 (Fig. 4D). These data suggested that aPD-L1 regulates the interaction between TAMs and TNBC cells.

\subsection{TNBC cells induces TAM/M2 polarization, and positive feedback promotes the malignant evolution of TNBC cells.}

To investigate whether TAM/M2 promotes the malignant progression of TNBC cells in co-culture system. We established cell co-culture system and performed wound healing and transwell analysis, the results confirmed that TAM/M2 promoted the migration of TNBC cells, while aPD-L1 reversed this phenomenon (Fig. 5A-B). To further investigated whether TAM/M2 directly induced angiogenesis of TNBC. Thus, we performed western blot analysis confirmed that TAM/M2 upregulated the expression of VEGF, MMP2, and 
MMP9 in TNBC cells, while aPD-L1 reversed the protein expression (Fig. 5C). In addition, TAM/M2 upregulated the expression of mesenchymal and stemness markers of TNBC cells, downregulated the expressions of epithelial markers, while APD-L1 reversed the EMT process and stemness of TNBC cells (Fig. 5D-E). These data suggested that APD-L1 inhibits the migration and angiogenesis of TNBC cells by regulating TAMs polarization mediated EMT process and cancer stemness.

\section{6 $\mathrm{APD}-\mathrm{L} 1$ regulates TAM/M2 polarization by downregulating STAT3 phosphorylation and preventing its entry into the nucleus.}

To further investigate the molecular mechanism by which aPD-L1 regulates TAMs polarization, we examined several immune-related pathways. The CREB-CEBP/ $\beta$, MAPK and STAT signaling pathways are all involved in TAM/M2 polarization ${ }^{[26,27]}$. We performed western blot analysis, and the results confirmed that IL-13 with or without aPD-L1 did not affect the expression of CEBP/ $\beta$. However, IL-13 upregulated the expression of $p$-ERK, p-STAT6 and p-STAT3, but the expression of p-ERK and p-STAT 6 had no effect after aPD-L1 treatment, while the expression of p-STAT3 was downregulated (Fig. 6A). IL-13 binding with its receptor promotes STAT3 phosphorylation to form a dimer into the nucleus ${ }^{[28]}$. We intend to investigate the location of STAT3 and p-STAT3 by IF staining assay, and the results confirmed that IL-13 had no effect on the expression of total STAT3, but its expression in the nucleus was increased, while aPD-L1 decreased the expression of STAT3 in the nucleus (Fig. 6B). IF staining analysis further confirmed that IL13 increased the expression of p-STAT3 and promoted its entry into the nucleus, while aPD-L1 decreased the expression of p-STAT3 and inhibited its entry into the nucleus (Fig. 6C).

\section{7 aPD-L1 prevents lung metastasis in vivo by targeting TAM/M2.}

We further investigate the effects of APD-L1 on lung metastasis in subcutaneous and intravenous models of TNBC. APD-L1 was dissolved in PBS and injected into tail vein at a dose of $10 \mathrm{~g} / \mathrm{kg}$, and the control group was treated with carrier (Fig. 7A). There was no significant difference in tumor growth and body weigh between the two groups in the TNBC subcutaneous modes (Fig. 7B-C, F). However, aPD-L1 reduced the number of metastatic nodes on lung tissue surface, $\mathrm{HE}$ staining of lung tissues further confirmed the presence of lung metastases (Fig. 7D-E). Similar results were obtained in the TNBC intravenous model (Fig. 7G-I). These data suggested that APD-L1 prevents lung metastasis in both subcutaneous and intravenous models of TNBC.

Subsequently, IHC and IF staining analysis confirmed that aPD-L1 reduced the number of CD206 in lung and tumor tissues, suggesting that aPD-L1 inhibits TAM/M2 polarization in vivo (Fig. 7J-K). IHC staining analysis further confirmed that APD-L1 upregulated the expression of E-Cadherin and downregulated the expression of Vimentin, VEGF and CD44 (Fig. 7L-O). These data suggested that aPD-L1 performs a substantial function in the evolution of TNBC. 


\section{Discussion}

The TME is complex and changeable, which is closely related to the occurrence and metastasis of tumor. TAMs is an important component of TME ${ }^{[29]}$. Cytokines such as IL-10 and TGF- $\beta$ secreted by TAM/M2 can inhibit tumor immune response and promote tumor development ${ }^{[30]}$. Tumor cells can release biomolecules into the TME and promote TAM/M2 polarization ${ }^{[31]}$. The number of TAM/M2 infiltrated in tumor tissues is related to the malignant biological behavior and poor prognosis of various solid tumors $^{[32]}$. The activation of immune regulatory site PD-1/PD-L1 plays a decisive role in the process of immune cell recognition. Blocking the expression of PD-L1 in host immune cells has more obvious tumor suppressive effects than blocking the expression of PD-L1 in tumor cells ${ }^{[22]}$. Targeting TAMs is an effective approach to modulate the activity of anti-PD-L1 agents for cancer therapy. ${ }^{[33]}$. This indicates that PD-L1 may play a role by directly action on macrophages. Our data suggest that APD-L1 reversed the expression of PD-L1, TAM/M2 marker CD206, and secretion of IL-10.

RAGE regulates TME by recruiting TAMs, thus promoting the progression and metastasis of TNBC ${ }^{[34]}$. However, the effects of blocking PD-1/PD-L1 on TAMs in the process of tumor metastasis remains to be further studied. We investigated the effects of aPD-L1 on tumor cells migration capability by regulating TAMs polarization in vitro. We believe that aPD-L1 reversed the migration of TNBC cells promoted by TAM/M2 polarization.

EMT contributes to enhance the capability of cancer cells to migrate and invade, which is critical in tumor metastasis. Many solid tumors have a large number of macrophages in the TME, which can promote tumor metastasis ${ }^{[35]}$. Cancer stemness originate from the fusion of TAM/M2 and breast cancer cells, these hybrid cells overexpress mesenchymal and cancer stemness markers ${ }^{[36]}$. Therefore, tumor cells are likely to become cancer stem cells after EMT process ${ }^{[37]}$. PD-1/PD-L1 induces immunosuppression and promotes EMT process of cancer cells. We investigated the effects of APD-L1 on EMT process and stemness of cancer cells by regulating TAMs polarization in vitro and in vivo. We believe that aPD-L1 reversed the EMT process and stemness of TNBC cells induced by TAM/M2 polarization.

Neovascularization in tumor tissues is an important condition for the metastasis of malignant tumors, and this process is regulated by various chemokines in $\mathrm{TME}^{[38]}$. Macrophages promote the secretion of VEGF by tumor cells to construct angiogenesis of local tumor tissues and provide nutrition for tumor growth and metastasis ${ }^{[39]}$. 4-HPR inhibits TAM/M2 phenotype, thereby inhibiting angiogenesis promotion $^{[40]}$. The expression of VEGF can promote metastasis of cancer cells ${ }^{[41]}$. MMP affects the expression of tumor cells adhesion molecules and promotes tumor cells beyond the basement membrane ${ }^{[42]}$. Our results confirmed that aPD-L1 downregulated VEGF, MMP2, and MMP9 expression by inhibiting TAM/M2 polarization. We believe that APD-L1 reversed the angiogenesis of TNBC induced by TAM/M2 polarization, which provides a new theoretical hypothesis for APD-L1 to inhibit tumor metastasis and angiogenesis. 
The conversion of macrophage phenotype depends on the activation of the signaling pathway response. The transcription factor $C E B P / \beta$ regulates the expression of EGR2 and participates in the polarization

process of macrophages ${ }^{[43]}$. Tumor tissue promotes TAM/M2 polarization and causes metastasis of lung cancer by upregulating ERK phosphorylation ${ }^{[44]}$. The STAT family regulates the biological behavior of tumor cells and immune cells through inflammatory mediators, and plays an important role in the process of inflammation-cancer transformation. STAT3 signal pathways play an important role in TAM/M2 polarization ${ }^{[45,46]}$. IL-13 binds to the IL-13 receptor on the surface of macrophages during the TAM/M2 polarization and initiates the intracellular tyrosine kinase phosphorylation cascade. STAT3 is activated in the cytoplasm after phosphorylation at Y705 and S727. Activated STAT3 can be transferred to the nucleus and promote TAM/M2 polarization. Overall, our data suggest that phosphorylation and nuclear translocation of STAT3 are involved in the regulation of TAM/M2 polarization by APD-L1.

\section{Conclusion}

Taken together, we indicated that APD-L1 inhibits TAM/M2 polarization in vitro and in vivo, which contributes to its anti-metastatic and anti-angiogenic function in TNBC. In addition, STAT3 plays a crucial role in the effects of aPD-L1 on TAM/M2 polarization (Fig. 8). Thus, PD-L1 may be a promising biomarker for the prognosis of TNBC patients and may provide a potential therapeutic target for TAMs in antimetastasis therapy.

\section{Abbreviations}

TNBC: Triple-negative breast cancer

TAMs: Tumor-associated macrophages

TAM/M2: M2-type macrophages

TAM/M1: M1-type macrophages

EMT: Epithelial-mesenchymal transition

HER2: Human epidermal growth factor receptor-2

TME: Tumor microenvironment

VEGF: Vascular endothelial growth factor

PD-1: Programmed death receptor-1

PD-L1: Programmed death ligand-1

HUVECs: Human umbilical vein endothelial cells 


\section{Declarations}

\section{Acknowledgments}

Not applicable.

\section{Author's contributions}

Ziqi Meng performed the data analyses and wrote the manuscript;Rui Zhang contributed significantly to analysis and manuscript preparation;Xuwei Wu performed the experiment;Meihua Zhang helped perform the analysis with constructive discussions. TiefengJin contributed to the conception of the study.All authors read and approved the final manuscript.

\section{Funding}

This study was supported by grants from the National Natural Science Foundation of China (No. 81960554).

\section{Availability of data and materials}

Data sharing not applicable to this article as no datasets were generated or analysed during the current study.

\section{Ethics approval}

"All applicable international, national, and/or institutional guidelines for the care and use of animals were followed."

\section{Consent for publication}

Not applicable.

\section{Competing interests}

The authors declare that they have no competing interests.

\section{References}


1. Zuo TT, Zheng RS, Zeng HM, et al. Female breast cancer incidence and mortality in China, 2013. Thorac Cancer. 2017;8(3):214-218.

2. Sung H, Ferlay J, Siegel RL, et al. Global cancer statistics 2020: GLOBOCAN estimates of incidence and mortality worldwide for 36 cancers in 185 countries.CA: a cancer journal for clinicians. 2021;71(3),209-249.

3. Shaikh S, Rasheed A. Predicting Molecular Subtypes of Breast Cancer with Mammography and Ultrasound Findings: Introduction of Sono-Mammometry Score. Radiol Res Pract. 2021;2021:6691958.

4. Jiagge EM, Ulintz PJ, Wong S, et al. Multiethnic PDX models predict a possible immune signature associated with TNBC of African ancestry. Breast Cancer Res Treat. 2021;186(2):391-401.

5. Manjunath M , Chou D Hary B . Triple-negative breast cancer: A run-through of features, classification and current therapies. Oncology Letters. 2021;22(1):512.

6. Li H, Fan X, Houghton J. Tumor microenvironment: the role of the tumor stroma in cancer. J Cell Biochem. 2007;101(4):805-815.

7. Emami F, Pathak S, Nguyen TT, et al. Photoimmunotherapy with cetuximab-conjugated gold nanorods reduces drug resistance in triple negative breast cancer spheroids with enhanced infiltration of tumor-associated macrophages. Journal of Controlled Release. 2021;329:645-664.

8. Mantovani A, Marchesi F, Malesci A, et al. Tumour-associated macrophages as treatment targets in oncology. Nature reviews Clinical oncology. 2017;14(7):399-416.

9. Adorno-Cruz V, Hoffmann A D, Liu X, et al. ITGA2 promotes expression of ACLY and CCND1 in enhancing breast cancer stemness and metastasis. Genes \& diseases. 2021;8(4):493-508.

10. Turajlic S, Swanton C. Metastasis as an evolutionary process. Science. 2016;352(6282):169-175.

11. Deng $\mathrm{Y}, \mathrm{Hu} \mathrm{JC}, \mathrm{He} \mathrm{SH}$, et al. Sphingomyelin synthase 2 facilitates M2-like macrophage polarization and tumor progression in a mouse model of triple-negative breast cancer. Acta Pharmacol Sin. 2021;42(1):149-159.

12. Zhang $X L$, Hu LP, Yang Q, et al. CTHRC1 promotes liver metastasis by reshaping infiltrated macrophages through physical interactions with TGF- $\beta$ receptors in colorectal cancer. Oncogene. 2021;40(23):3959-3973.

13. Tang $C$, Lei $X$, Xiong $L$, et al. HMGA1B/2 transcriptionally activated-POU1F1 facilitates gastric carcinoma metastasis via CXCL12/CXCR4 axis-mediated macrophage polarization. Cell Death Dis. 2021;12(5):422.

14. Pastushenko I, Blanpain C. EMT Transition States during Tumor Progression and Metastasis. Trends Cell Biol. 2019;29(3):212-226.

15. Yang C, Dou R, Wei C, et al. Tumor-derived exosomal microRNA-106b-5p activates EMT-cancer cell and M2-subtype TAM interaction to facilitate CRC metastasis. Mol Ther. 2021;29(6):2088-2107.

16. Clevers H. The cancer stem cell: premises, promises and challenges. Nat Med. 2011;17(3):313-319. 
17. Chen $Y$, Tan W, Wang C. Tumor-associated macrophage-derived cytokines enhance cancer stem-like characteristics through epithelial-mesenchymal transition. Onco Targets Ther. 2018;11:3817-3826.

18. Larionova I, Kazakova E, Gerashchenko T, et al. New Angiogenic Regulators Produced by TAMs: Perspective for Targeting Tumor Angiogenesis. Cancers (Basel). 2021;13(13):3253.

19. Gatalica Z, Snyder C, Maney T, et al. Programmed cell death 1 (PD-1) and its ligand (PD-L1) in common cancers and their correlation with molecular cancer type. Cancer Epidemiol Biomarkers Prev. 2014;23(12):2965-2970.

20. Cimino-Mathews A, Foote JB, Emens LA. Immune targeting in breast cancer. Oncology (Williston Park). 2015;29(5):375-385.

21. Moser JC, Hu-Lieskovan S. Mechanisms of Resistance to PD-1 Checkpoint Blockade. Drugs. 2020;80(5):459-465.

22. Noguchi T, Ward JP, Gubin MM, et al. Temporally Distinct PD-L1 Expression by Tumor and Host Cells Contributes to Immune Escape. Cancer Immunol Res. 2017;5(2):106-117.

23. Juneja VR, McGuire KA, Manguso RT, et al. PD-L1 on tumor cells is sufficient for immune evasion in immunogenic tumors and inhibits CD8 T cell cytotoxicity. J Exp Med. 2017;214(4):895-904.

24. Fu LQ, Du WL, Cai MH, et al. The roles of tumor-associated macrophages in tumor angiogenesis and metastasis. Cell Immunol. 2020;353:104119.

25. Wang W, Zhao Y, Yao S, et al. Nigericin Inhibits Epithelial Ovarian Cancer Metastasis by Suppressing the Cell Cycle and Epithelial-Mesenchymal Transition. Biochemistry (Mosc). 2017;82(8):933-941.

26. Lawrence T, Natoli G. Transcriptional regulation of macrophage polarization: enabling diversity with identity. Nat Rev Immunol. 2011;11(11):750-761.

27. Neamatallah T. Mitogen-Activated Protein Kinase Pathway: A Critical Regulator in Tumor-associated Macrophage Polarization. J Microsc Ultrastruct. 2019;7(2):53-56.

28. LaPorte SL, Juo ZS, Vaclavikova J, et al. Molecular and structural basis of cytokine receptor pleiotropy in the interleukin-4/13 system. Cell. 2008;132(2):259-272.

29. Boutilier AJ, Elsawa SF. Macrophage Polarization States in the Tumor Microenvironment. Int J Mol Sci. 2021;22(13):6995.

30. Ma YY, He XJ, Wang HJ, et al. Interaction of coagulation factors and tumor-associated macrophages mediates migration and invasion of gastric cancer. Cancer Sci. 2011;102(2):336-342.

31. Mills CD, Lenz LL, Harris RA. A Breakthrough: Macrophage-Directed Cancer Immunotherapy. Cancer Res. 2016;76(3):513-516.

32. Sami E, Paul BT, Koziol JA, et al. The Immunosuppressive Microenvironment in BRCA1-IRISOverexpressing TNBC Tumors Is Induced by Bidirectional Interaction with Tumor-Associated Macrophages. Cancer Res. 2020;80(5):1102-1117.

33. Santoni M, Romagnoli E, Saladino T, et al. Triple negative breast cancer: Key role of TumorAssociated Macrophages in regulating the activity of anti-PD-1/PD-L1 agents. Biochim Biophys Acta Rev Cancer. 2018;1869(1):78-84. 
34. Nasser MW, Wani NA, Ahirwar DK, et al. RAGE mediates S100A7-induced breast cancer growth and metastasis by modulating the tumor microenvironment. Cancer Res. 2015;75(6):974-985.

35. Nieto MA, Huang RY, Jackson RA, et al. EMT: 2016. Cell. 2016;166(1):21-45.

36. Li B, Lu Y, Yu L, et al. miR-221/222 promote cancer stem-like cell properties and tumor growth of breast cancer via targeting PTEN and sustained Akt/NF-KB/COX-2 activation. Chem Biol Interact. 2017;277:33-42.

37. Zhou Y, Chen D, Qi Y, et al. Evaluation of expression of cancer stem cell markers and fusion gene in synovial sarcoma: Insights into histogenesis and pathogenesis. Oncol Rep. 2017;37(6):3351-3360.

38. Samples J, Willis M, Klauber-Demore N. Targeting angiogenesis and the tumor microenvironment. Surg Oncol Clin N Am. 2013;22(4):629-639.

39. Barnett FH, Rosenfeld M, Wood M, et al. Macrophages form functional vascular mimicry channels in vivo. Sci Rep. 2016;6:36659.

40. Dong R, Gong $Y$, Meng W, et al. The involvement of $M 2$ macrophage polarization inhibition in fenretinide-mediated chemopreventive effects on colon cancer. Cancer Lett. 2017;388:43-53.

41. Wang H, Yung MMH, Ngan HYS, et al. The Impact of the Tumor Microenvironment on Macrophage Polarization in Cancer Metastatic Progression. Int J Mol Sci. 2021;22(12):6560.

42. Wiśniowski T, Bryda J, Kurzepa J, et al. The role of matrix metalloproteinases in pathogenesis of human bladder cancer. Acta Biochim Pol. 2021;10.18388/abp.2020_5600.

43. Veremeyko T, Yung AWY, Anthony DC, et al. Early Growth Response Gene-2 Is Essential for M1 and M2 Macrophage Activation and Plasticity by Modulation of the Transcription Factor CEBP $\beta$. Front Immunol. 2018;9:2515.

44. Sami E, Paul BT, Koziol JA, et al. The Immunosuppressive Microenvironment in BRCA1-IRISOverexpressing TNBC Tumors Is Induced by Bidirectional Interaction with Tumor-Associated Macrophages. Cancer Res. 2020;80(5):1102-1117.

45. Xu J, Zhang J, Zhang Z, et al. Hypoxic glioma-derived exosomes promote M2-like macrophage polarization by enhancing autophagy induction. Cell Death Dis. 2021;12(4):373.

46. Qian M, Wang S, Guo X, et al. Hypoxic glioma-derived exosomes deliver microRNA-1246 to induce M2 macrophage polarization by targeting TERF2IP via the STAT3 and NF-KB pathways. Oncogene. 2020;39(2):428-442.

\section{Figures}



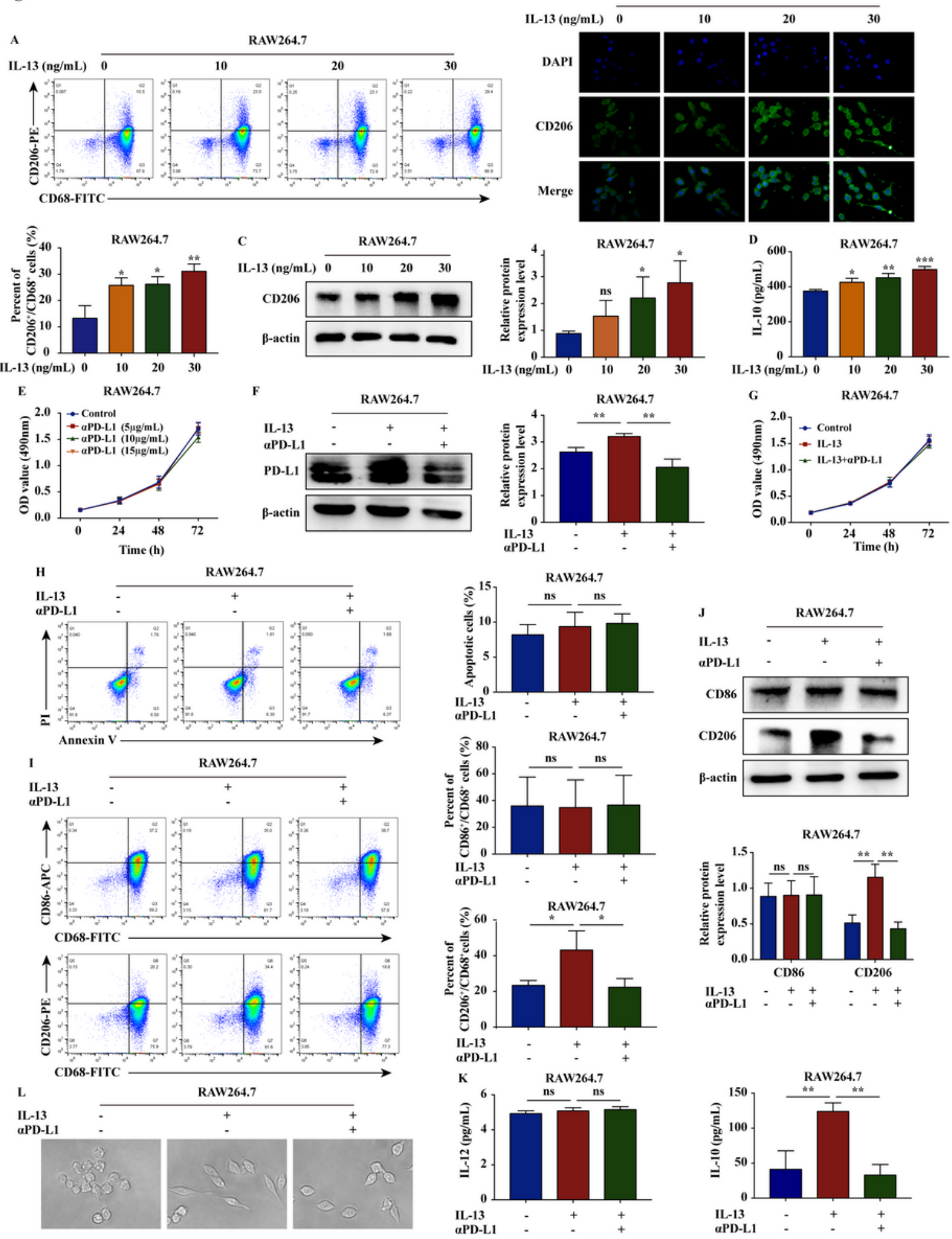

Figure 1

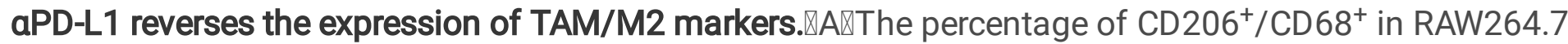
cells treated with IL-13 $(0,10,20,30 \mathrm{ng} / \mathrm{mL})$ was confirmed by flow cytometry assay (Q2 regional). $\triangle \mathrm{B}-\mathrm{C} \mathbb{\mathrm { V }}$ The expression of CD206 in RAW264.7 cells treated with IL-13 was confirmed by IF staining (original magnification, 400x) and western blot assays, and the markers were all expressed in the cytoplasm. (D) The secretion of IL-10 in RAW264.7 cells treated with IL-13 was confirmed by ELISA assay. (E) The 
viability of RAW264.7 cells treated with aPD-L1 $(0,5,10,15 \mu \mathrm{g} / \mathrm{mL})$ was confirmed by MTT assay. $(\mathrm{F})$ The protein expression of PD-L1 in RAW264.7 cells treated with IL-13 $(30 \mathrm{ng} / \mathrm{mL})$ and/or aPD-L1 $(15 \mu \mathrm{g} / \mathrm{mL})$ was confirmed by western blot assay. (G) The proliferation capacity of RAW264.7 cells treated with IL-13 and/or APD-L1 was measured by MTT assay. $(H)$ The apoptosis capacity of RAW264.7 cells treated with IL-13 and/or aPD-L1 was confirmed by flow cytometry assay. (I-J) The expression of CD86 and CD206 in RAW264.7 cells treated with IL-13 and/or aPD-L1 was confirmed by flow cytometry and western blot assays. (K) The secretion of IL-12 and IL-10 in RAW264.7 cells treated with IL-13 and/or aPD-L1 was confirmed by ELISA assay. (L)The morphology of RAW264.7 cells treated with IL-13 and/or aPD-L1was observed by microscope. 
Figure 2

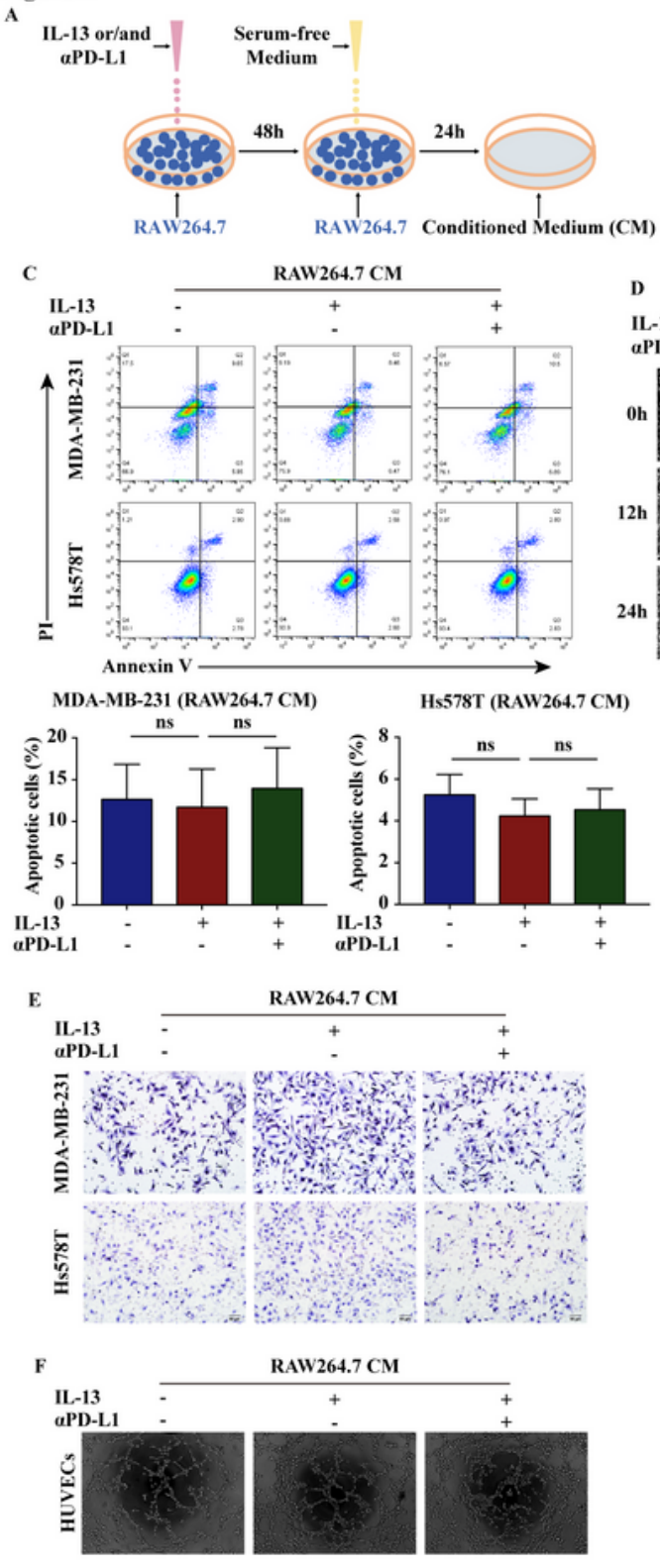

h
B MDA-MB-231 (RAW264.7 CM)

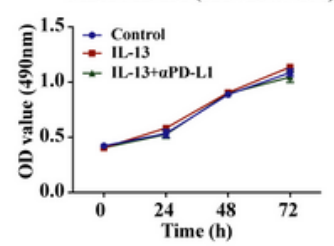

MDA-MB-231 (RAW264.7 CM)
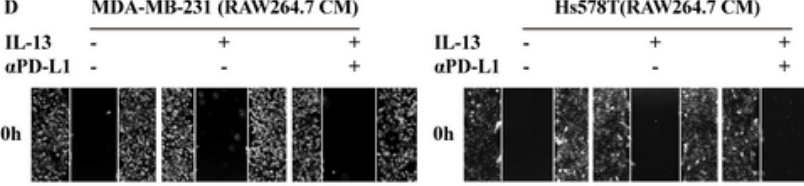

oh

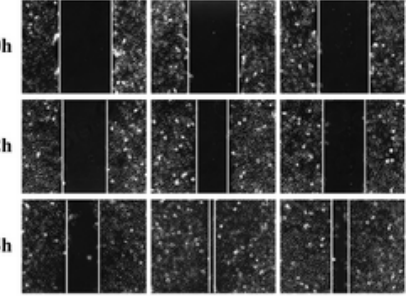

@ MDA-MB-231 (RAW264.7 CM)
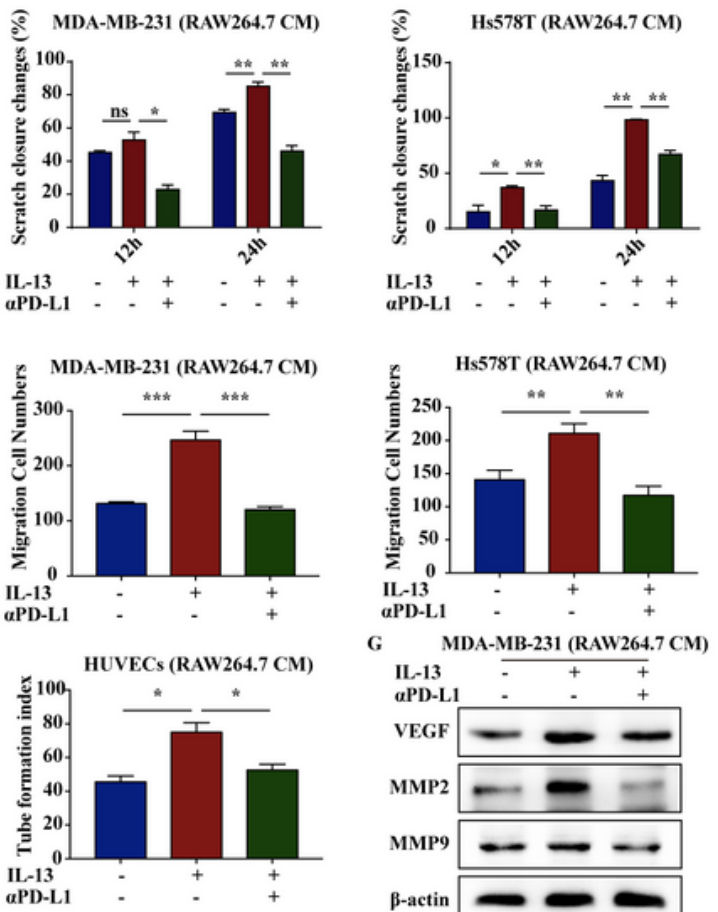

G MDA-MB-231 (RAW264.7 CM)
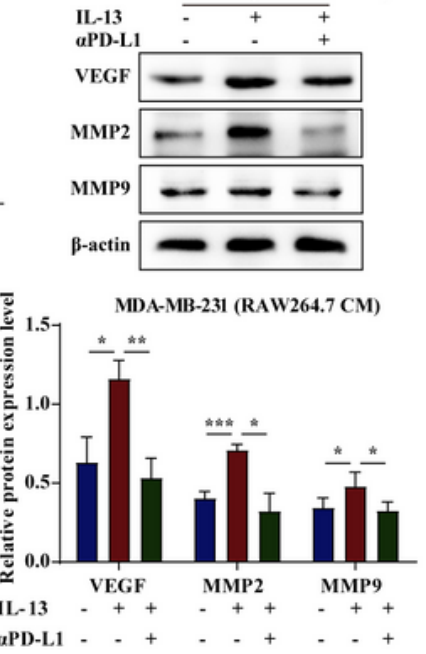

Figure 2

aPD-L1 inhibits the migration and angiogenesis of TNBCpromoted by TAM/M2. (A) The schematic diagram of RAW264.7 cells CM preparation. $\triangle \mathrm{B}-\mathrm{C} \otimes \mathrm{T}$ The proliferation and apoptosis capacity of MDA-MB231 and Hs578T cells treated with CM was confirmed by MTT and flow cytometry assays. (D-E) The migration capacity ofMDA-MB-231 and Hs578T cells treated with CM was confirmed by wound healing and transwell assays(original magnification, 200x). (F) The microtubule formation capacity of HUVECs 
treated with CM was confirmed by endothelial tube formation assay. (G) The protein expression of VEGF, MMP2, and MMP9 in MDA-MB-231 and Hs578T cells treated with CM was confirmed by western blot assay.

Figure 3
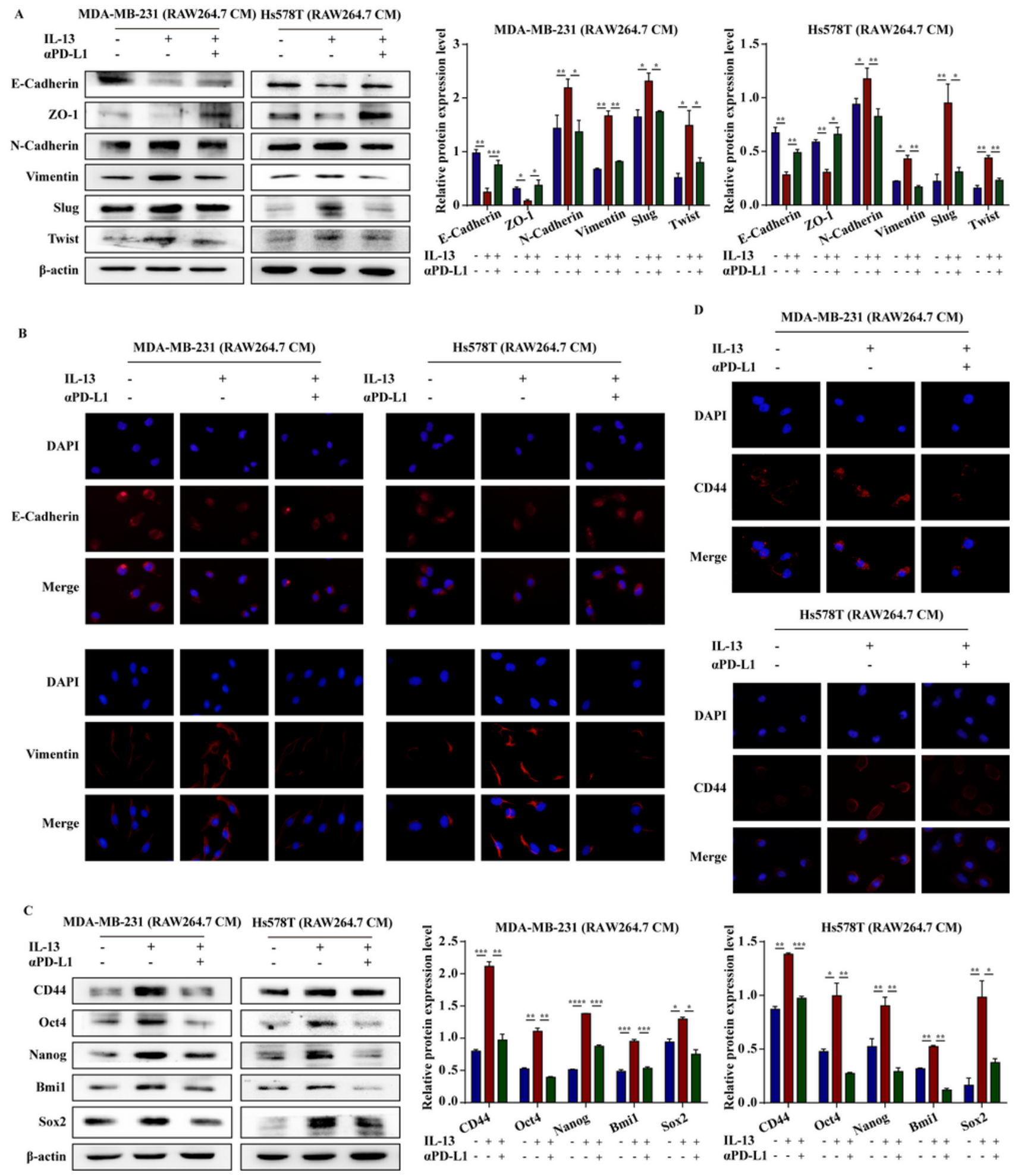

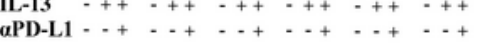
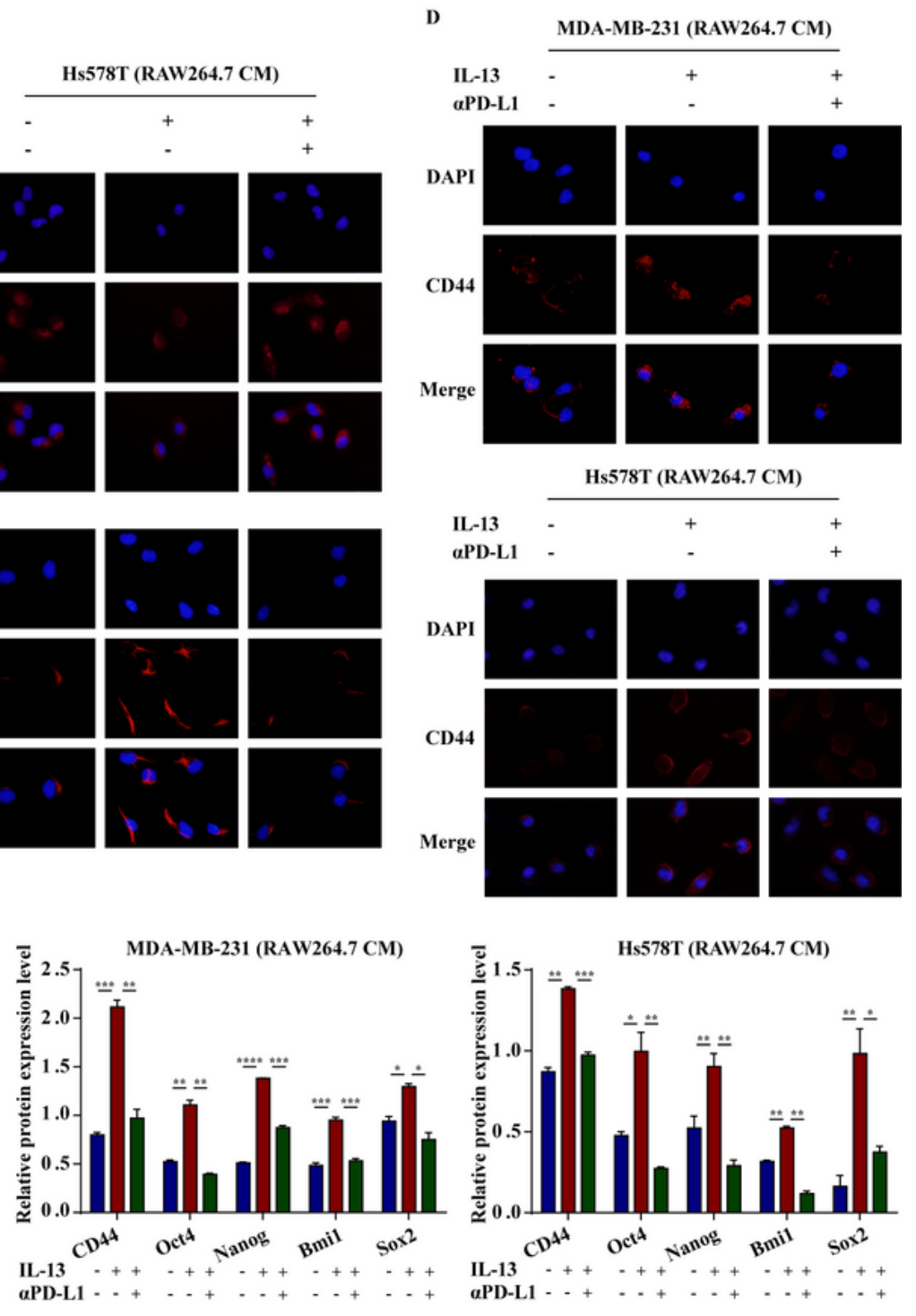

Figure 3 
aPD-L1 inhibits the EMT process and stemness of TNBC promoted by TAM/M2. (A) The protein expression of EMT markers (E-Cadherin, ZO-1, N-Cadherin, Vimentin, Slug, and Twist) in MDA-MB-231 and Hs578T cells treated with CM was confirmed by western blot assay. (B) The expression of E-Cadherin and Vimentin in MDA-MB-231 and Hs578T cells treated with CM was confirmed by IF staining assay, and the markers were all expressed in the cytoplasm (original magnification, 400x). (C) The protein expression of stemness markers (CD44, Oct4, Nanog, Bmi1, and Sox2) in MDA-MB-231 and Hs578T cells treated with CM was confirmed by western blot assay. (D) The expression of CD44 in MDA-MB-231 and Hs578T cells treated with CM was confirmed by IF staining assay, and the markers were all expressed in the cytoplasm (original magnification, 400x). 
Figure 4

\begin{tabular}{|l|}
\hline Transwell co-culture system \\
\hline
\end{tabular}
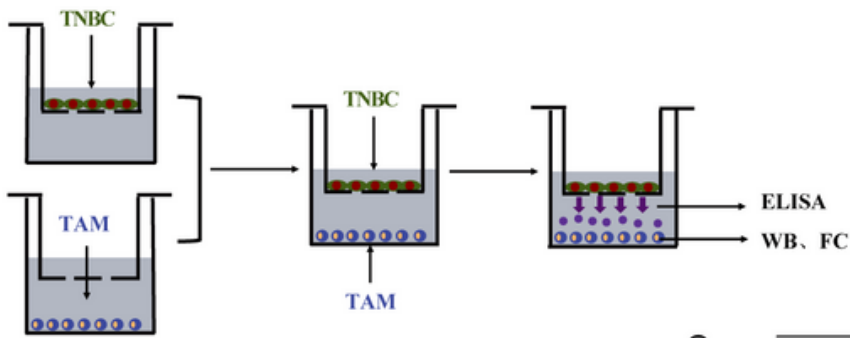

B

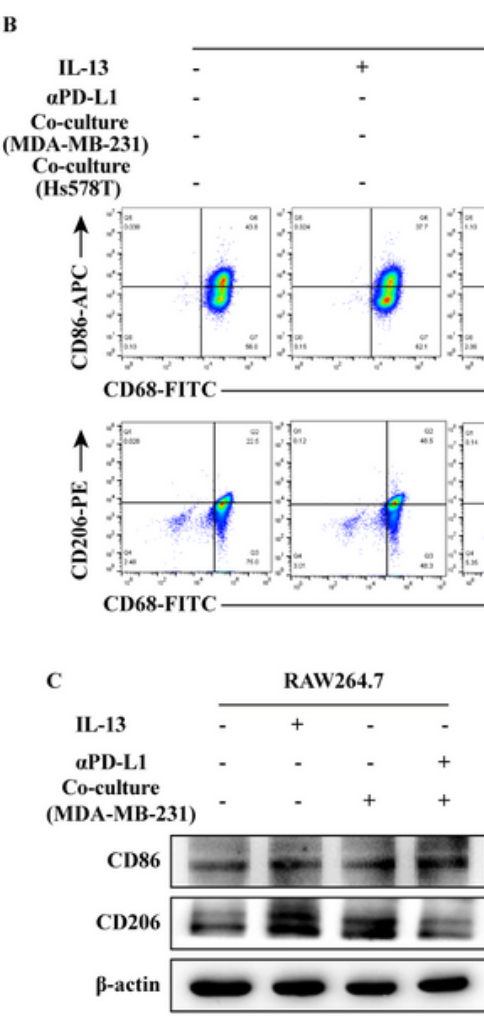

RAW264.7

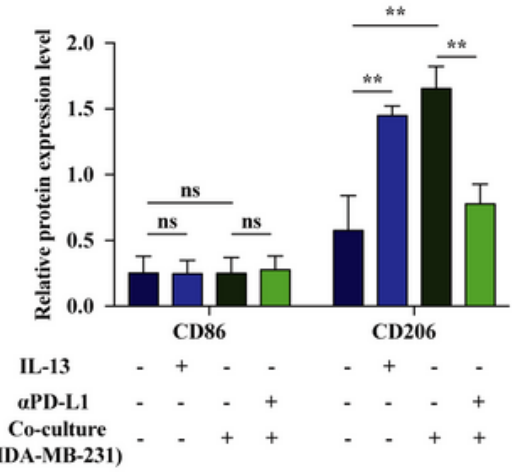

RAW264.7
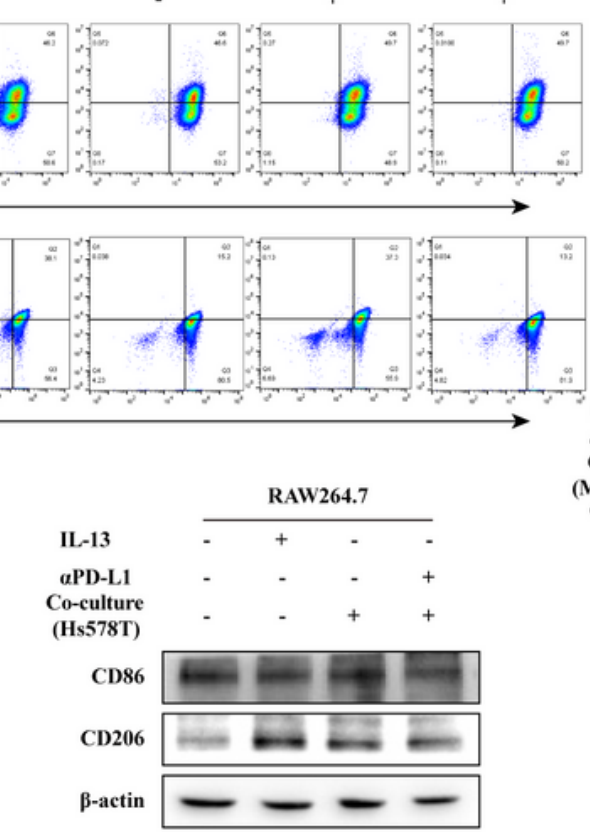

RAW264.7

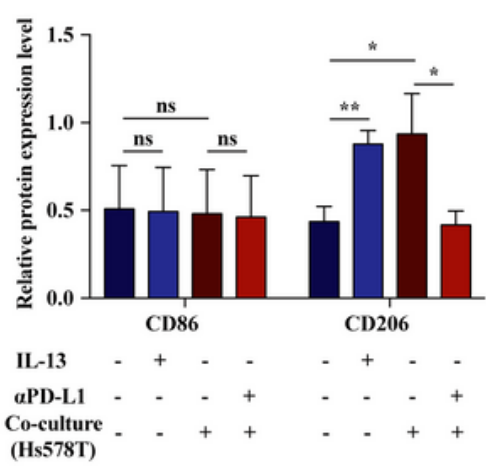

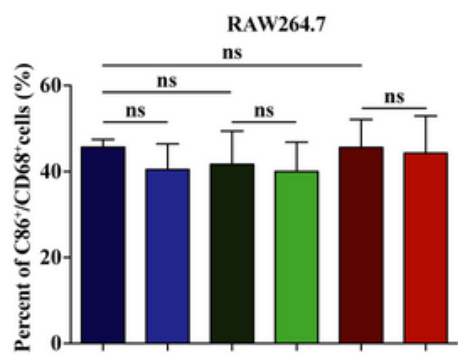

RAW264.7
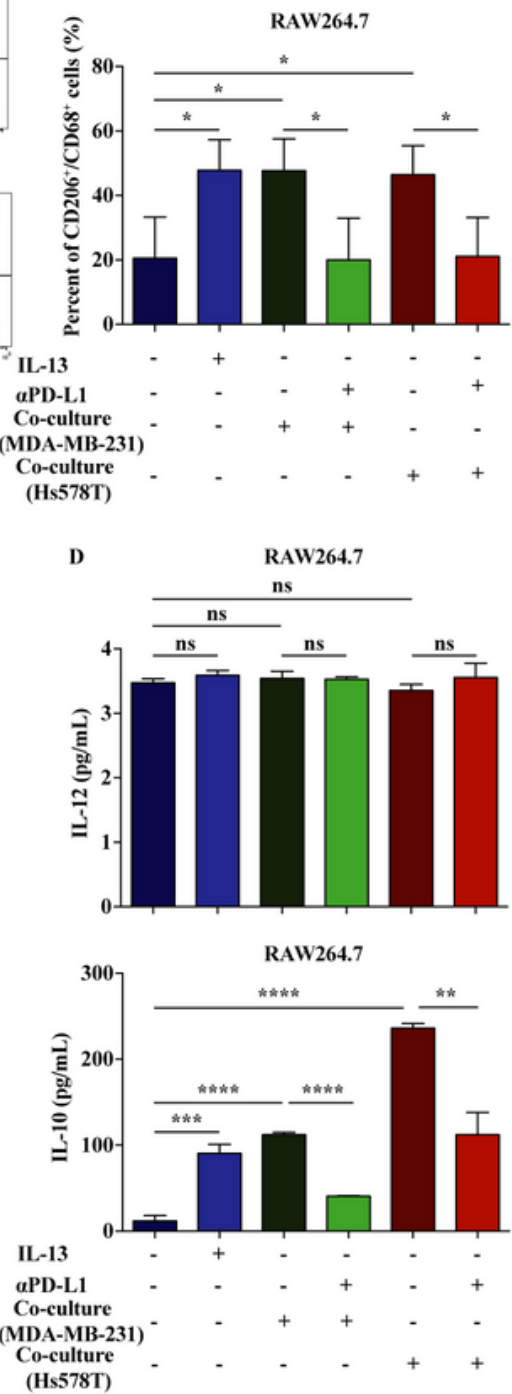

Figure 4

aPD-L1 reverses TAM/M2 polarization in co-culture of TNBC cells and TAMs.(AXThe schematic diagram of TNBC cells and TAMs co-culture system. (B) RAW264.7 cells were co-cultured with MDA-MB-231 and Hs578T cells, respectively. The percentage of $\mathrm{CD} 86^{+} / \mathrm{CD} 68^{+}$and $\mathrm{CD} 206^{+} / \mathrm{CD} 68^{+}$treated with or without aPD-L1 in co-culture system was confirmed by flow cytometry assay. (C) The protein expression of CD86 and CD206 treated with or without aPD-L1 in co-culture system was confirmed by western blot assay. (D) 
The secretion of IL-12 and IL-10 treated with or without APD-L1 in co-culture system was confirmed by ELISA assay.

Figure 5
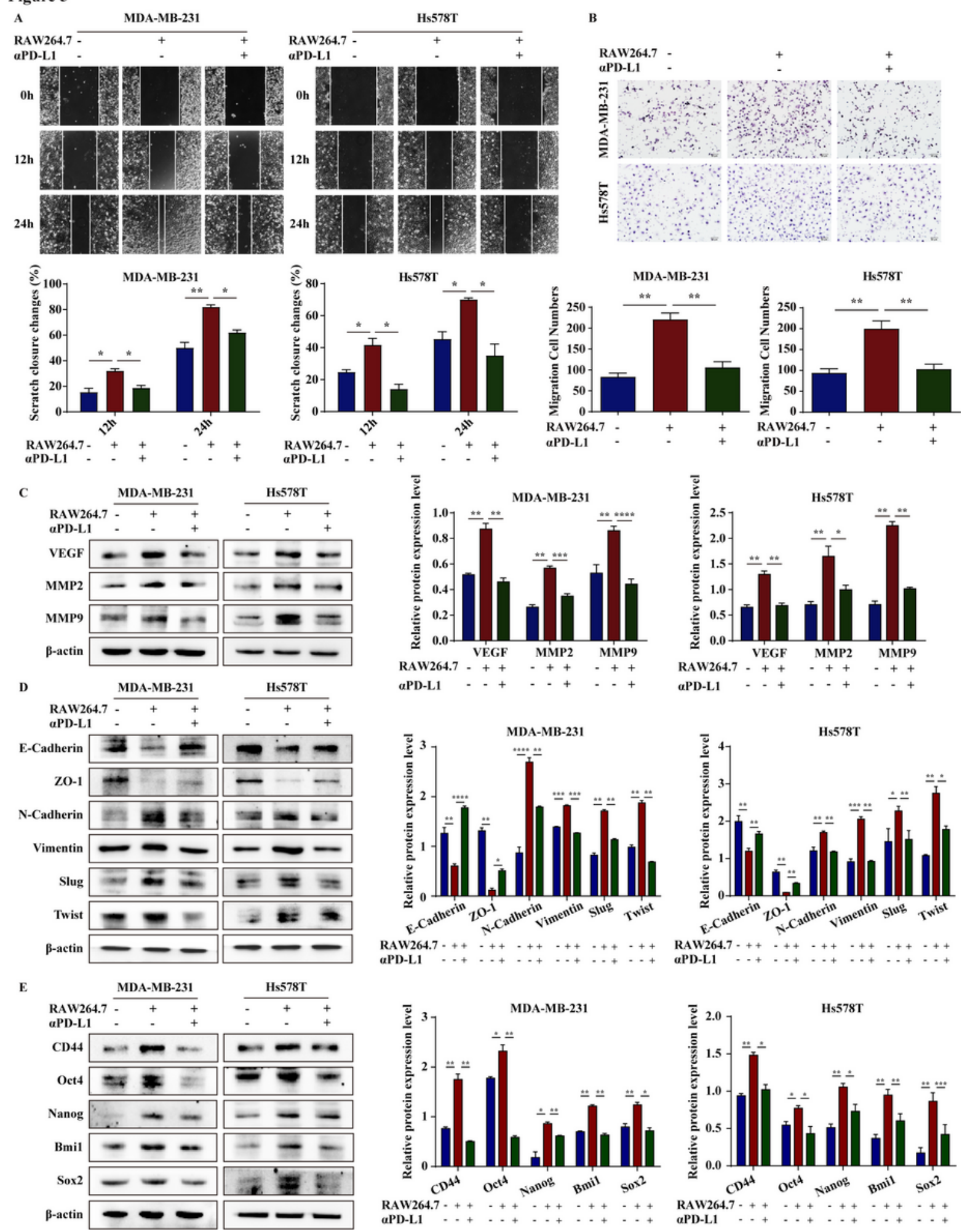

Figure 5

aPD-L1 reverses TNBC malignant evolution in co-culture of TNBC cells and TAMs. $\triangle A-B \otimes T$ The migration capacity of MDA-MB-231 and Hs578T cells treated with or without APD-L1 in co-culture system was 
confirmed by wound healing and transwell assays (original magnification, 200x). (C) The protein expression of VEGF, MMP2, and MMP9 in MDA-MB-231 and Hs578T cells with or without APD-L1 in coculture system was confirmed by western blot assay. (D) The protein expression of EMT markers (ECadherin, ZO-1, N-Cadherin, Vimentin, Slug, and Twist) in MDA-MB-231 and Hs578T cells with or without APD-L1 in co-culture system was confirmed by western blot assay. (E) The protein expression of stemness markers (CD44, Oct4, Nanog, Bmi1 and Sox2) in MDA-MB-231 and Hs578T cells with or without APD-L1 in co-culture system was confirmed by western blot assay.

Figure 6

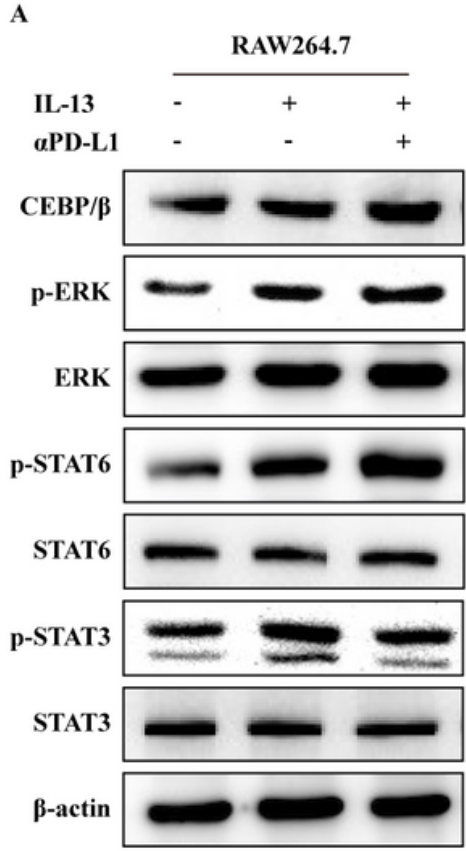

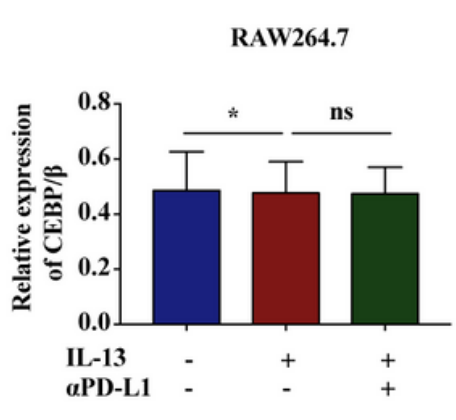

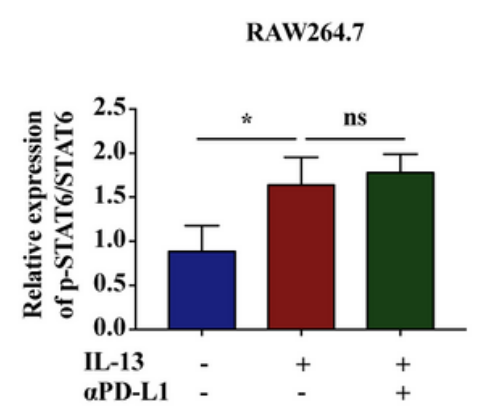

RAW264.7
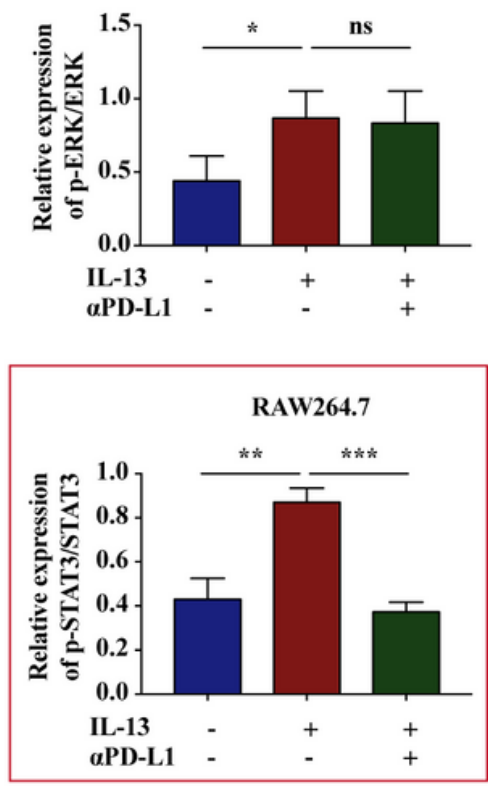

B

IL-13

aPD-L1
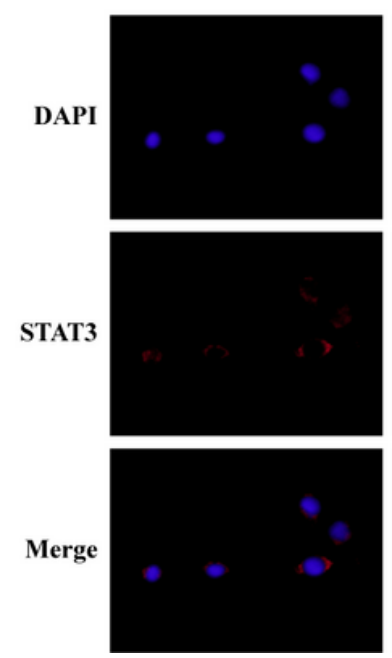

RAW264.7
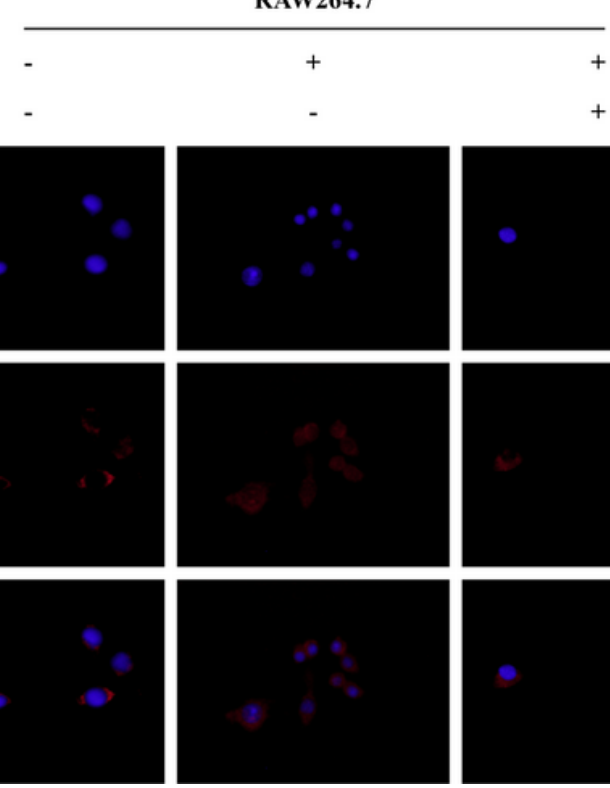
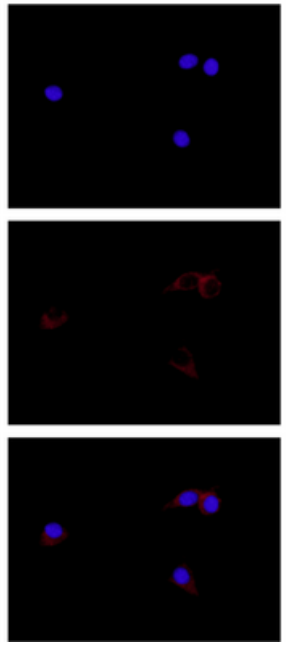

C

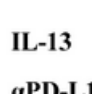

aPD-L1

DAPI

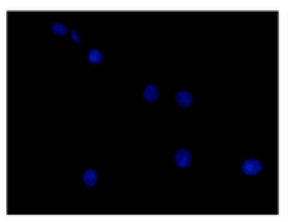

p-STAT3

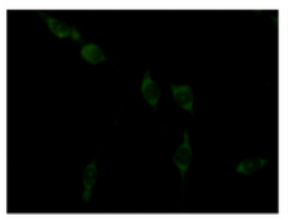

Merge

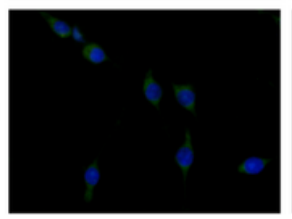

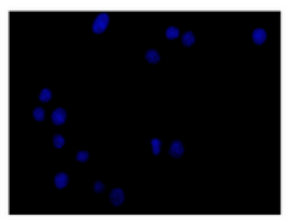

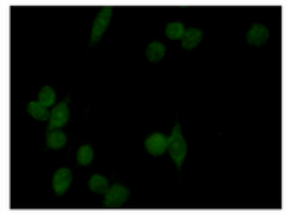

RAW264.7

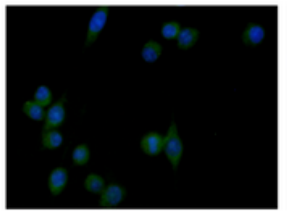

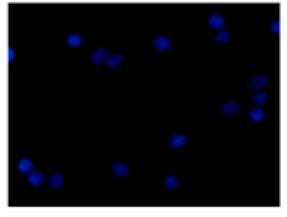
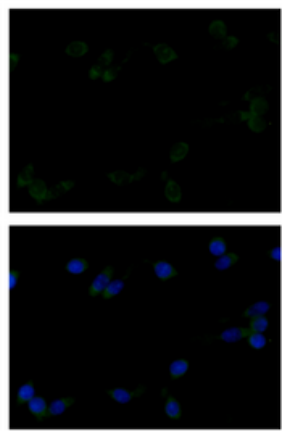

Figure 6 


\section{aPD-L1 inhibits TAM/M2 polarization via prevention of STAT3 phosphorylation and nuclear}

translocation. (A) The protein expression of CEBP/ $\beta$, p-ERK, ERK, p-STAT6, STAT6,p-STAT3, and STAT3 in RAW264.7 cells treated with IL-13 and/or aPD-L1 was confirmed by western blot assay. (B-C) The expression and nuclear translocation of STAT3 and p-STAT3 in RAW264.7 cells treated with IL-13 and/or aPD-L1 was confirmed by IF staining assay (original magnification, 400×).
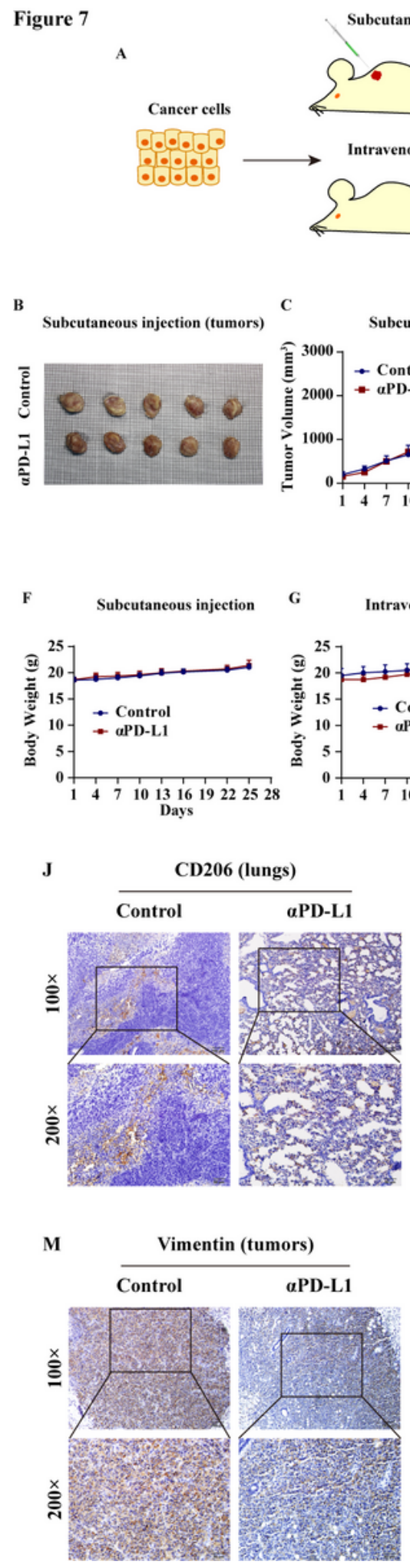

K

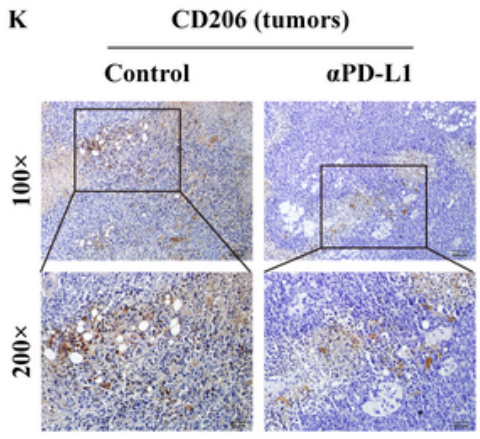

$\mathbf{N}$

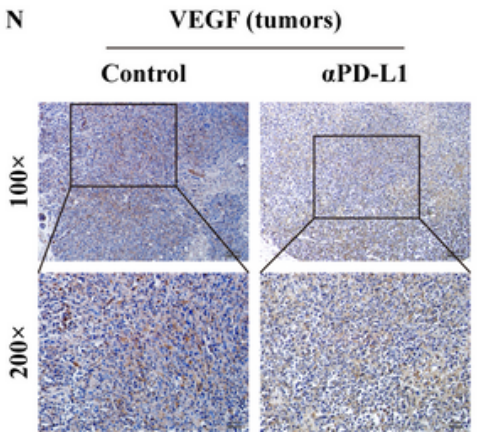

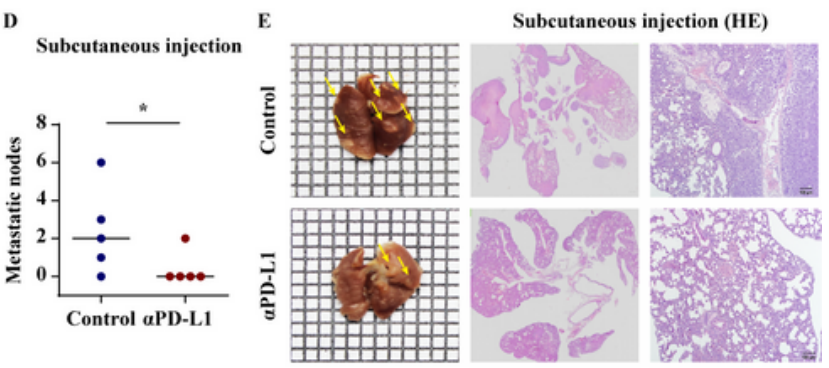

H

Intravenous injection

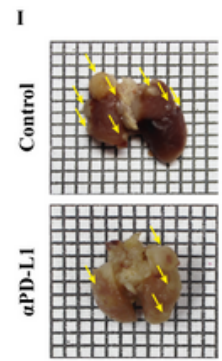

Intravenous injection (HE)

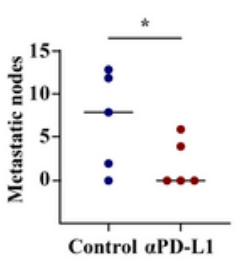

L

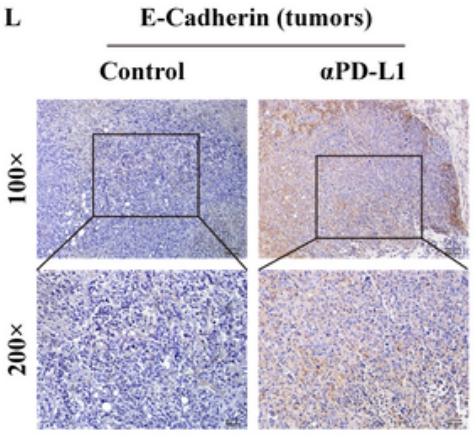

o

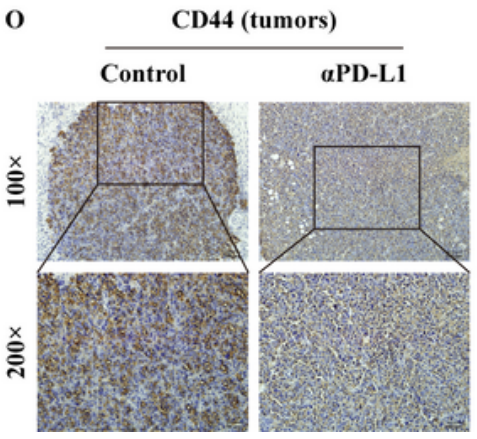

Figure 7 
aPD-L1 inhibits lung metastasis in vivo. $\triangle A \otimes$ The schematic diagram of animal assays. (B-C) The tumor volume in subcutaneous transplanted mice were recorded over time in the process of the animal assay. $(D, H)$ The number of lung metastatic nodules was determined. (E) Lung metastases of TNBC cells were subcutaneously injected. Lung tissue images, HE scan images, and HE magnification images (original magnification, 200x) were shown respectively. (F-G) The body weight in subcutaneous and intravenous transplanted mice was recorded over time in the process of the animal assay. (I) Lung metastases of TNBC cells were intravenously injected. Lung tissue images, HE scan images, and HE magnification images (original magnification, 200x) were shown respectively.(J-K) The expression of CD206 in the lung and tumor tissues was confirmed by IHC and IF staining assays. (L-O) The expression of E-Cadherin, Vimentin, VEGF, and CD44 in the tumors tissues was confirmed by IHC staining.

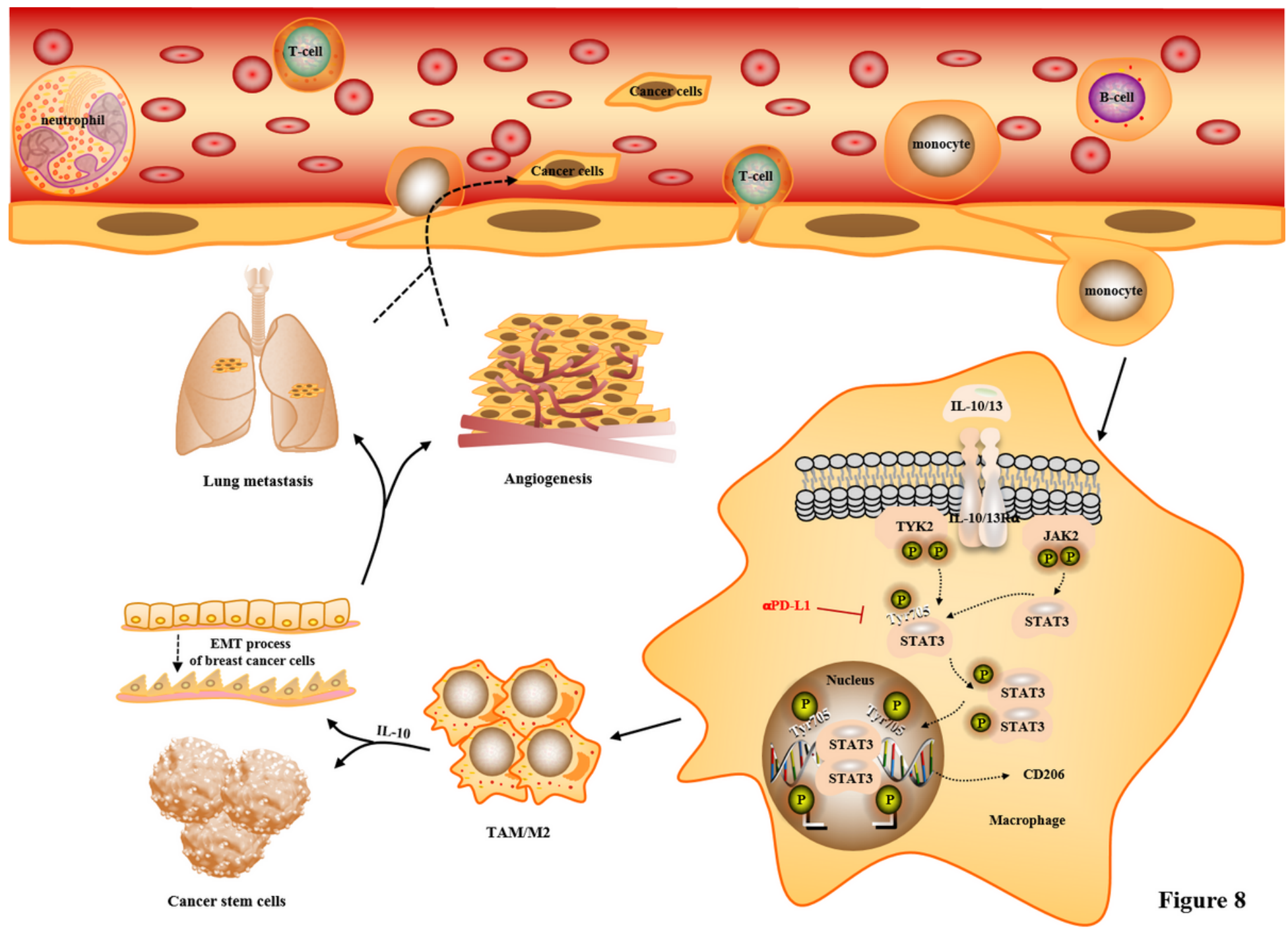

Figure 8

Schematic overview on the mechanisms by whichaPD-L1 regulates TAMs polarization and promotes breast cancer malignant progression. 